\title{
Prostatic meningeal carcinomatosis presenting as delirium tremens
}

\author{
Jeffrey B Rubins, Manuel J Guzman-Paz
}

\begin{abstract}
Summary
We report an unusual case of prostatic carcinomatous meningitis and remind clinicians to maintain a high index of suspicion of meningeal involvement when patients with advanced prostatic cancer present with cerebral symptoms, back pain, or neurologic findings. The diagnosis may require repeated cytologic examinations of cerebrospinal fluid, and immunocytochemical stains should be considered to confirm a prostatic source if malignant cells are identified. Androgen ablative therapy may give prolonged remissions, especially in patients with previously untreated tumours.
\end{abstract}

Keywords: prostatic neoplasms, meningeal carcinomatosis, delirium, leuprolide

Prostate carcinoma will be diagnosed in an estimated 300000 Americans, and advanced prostate carcinoma will account for over 41 000 deaths in 1996. In advanced disease, prostate carcinoma typically metastasizes to local lymph nodes and bone, with metastases to visceral organs being much less common. However, with the advent of hormonal therapy, patients with advanced stages of prostate carcinoma are surviving longer, and more unusual presentations of advanced prostate carcinoma may become more common. We report a case of unsuspected prostatic carcinomatous meningitis mimicking delirium tremens and review the existing literature on this unusual complication of advanced prostate cancer.

Veterans Affairs

Medical Center and University of Minnesota, Minneapolis, MN 55417, USA

Department of Medicine

JB Rubins

Department of Laboratory Services MJ Guzman-Paz

Correspondence to JB Rubins, MD, Pulmonary (111N), VA Medical Center, One Veterans Drive, Minneapolis, MN 55417, USA

Accepted 28 May 1997 (measured by ELISA, normal < 4) for the preceding six months.
Physical examination and laboratory testing revealed only mild dehydration, and the patient was treated conservatively with rehydration and observation for a presumptive viral illness. On the second day of admission, the patient became delirious and tremulous, with tachycardia and hypertension, and was treated for ethanol withdrawal delirium with intravenous lorazepam. His course was complicated by prolonged confusion and tremulousness over the next six days, and by worsening of his obstructive airways disease, requiring a short course of systemic steroids and inhaled bronchodilators. Subsequently, his condition rapidly improved over the next week, and he was discharged to a supervised facility for rehabilitation and counselling regarding his ethanol abuse.

He was re-admitted 36 hours after discharge with acute delirium and tremulousness, tachycardia, and hypertension. Neurological examination was limited by the patient's delirium but did not reveal focal deficits. Because he had not ingested ethanol during his previous hospital admission or since discharge, other causes for his delirium such as subdural haematoma and meningitis were sought. Computed tomography (CT) of the head with intravenous contrast did not reveal any evidence of acute haemorrhage or metastases. Examination of his cerebrospinal fluid (CSF) after lumbar puncture showed an elevated total protein of $397 \mathrm{mg} / \mathrm{dl}$ (normal 15-50) and white blood cell count of 64 cells $/ \mathrm{mm}^{3}$. Microbiological stains and cultures were negative. Cytologic examination revealed cohesive aggregates of malignant cells, which stained positive for cytokeratin (AE1/AE3, 1:80, Biogenex, San Ramon, CA; Cam 5.2, 1:20, Becton-Dickinson, San Jose, CA; MAK-6, 1:50, Triton Biosciences, Alameda, CA), epithelial membrane antigen (1:100, Dako, Carpinteria, CA), prostate-specific antigen (1:5000, Dako) and prostatic acid phosphatase (1:5000, Dako) (figure 1). Subsequent evaluation included a bone scan showing new areas of increased technetium uptake in the parietal skull, ribs, and spine compared to a previous study four months earlier. Re-examination by thin-cut head CT scan confirmed a blastic metastatic focus in the right parietal skull. Initiation of intravenous methylprednisolone $100 \mathrm{mg}$ every $6 \mathrm{~h}$ initially improved the patient's delirium, tachycardia, and hypertension, allowing the patient and his family to discuss further treatment options. Chemotherapy, radiation therapy, or further hormonal 


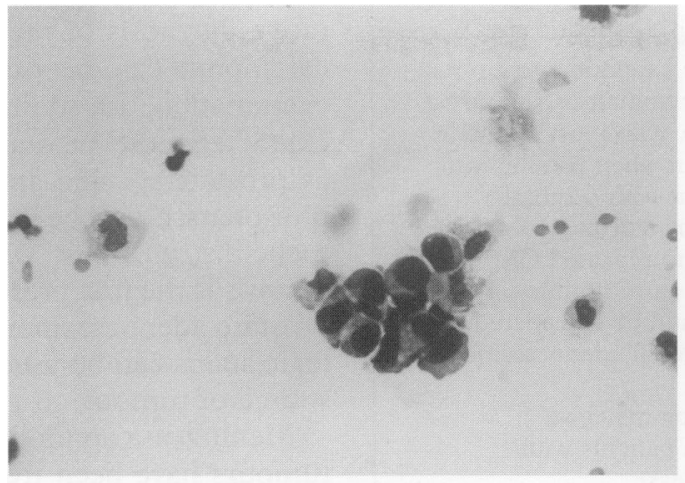

A

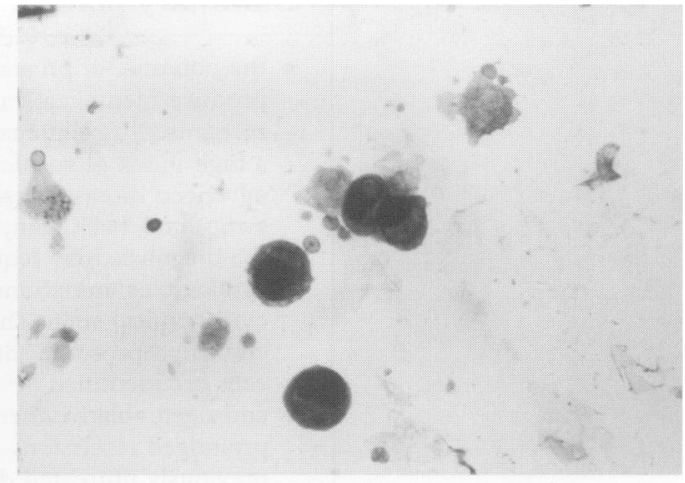

B

Figure 1 Cerebrospinal fluid cytology. (A) Group of malignant cells (Wright stain, original magnification X400); (B) positive staining of tumour cells with prostate-specific antigen (Immunoperoxidase, original magnification X400)

therapies were declined by the patient and his family, and he died of pneumonia in a hospice three weeks after the diagnosis was made.

At autopsy, a high-grade small acinar adenocarcinoma showing effects of leuprolide therapy was identified in the prostate. Osteoblastic metastases to vertebral column, rib, and skull, and metastases to lung were also noted. Extensive infiltration of the leptomeninges was identified at all levels of the neuraxis, including the cerebrum, cerebellum, pituitary, brainstem, cranial nerve roots (III, X, and XII), and spinal cord (figure 2). No dural, intraparenchymal, intraventricular or ependymal metastases were identified.

\section{Discussion}

Prostate carcinoma is an extremely rare cause of meningeal carcinomatosis. Only six welldocumented cases of prostatic meningeal carcinomatosis have been reported in the literature, ${ }^{1-3}$ with an additional two cases mentioned in one large review. ${ }^{4}$ Furthermore, metastatic prostate carcinoma infiltrating the leptomeninges has only been documented in one previous autopsy. ${ }^{2}$ Consistent with our case report, the intervals between the diagnosis of the primary tumour and the first positive CSF cytology in these cases ranged from 11 to

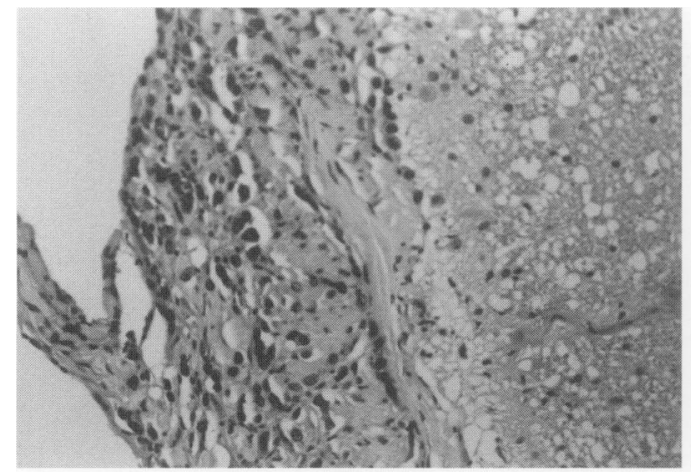

A

Figure 2 Malignant invasion of leptomeninges. (A) Diffuse infiltration of the leptomeninges by malignant cells (Hematoxylin and eosin, original magnification X200); (B) involvement of cranial nerve roots (Hematoxylin and eosin, original magnification $\mathrm{X} 100$ )
76 months, with an average of 40 months. Also, our patient's survival of 21 days was typical of the reported cases, who survived from three days to more than 16 months after diagnosis, with a median survival of 90 days.

Although prostate carcinoma is rarely identified in case series of meningeal carcinomatosis, the incidence of meningeal carcinomatosis among cases of prostate cancer is unknown because the diagnosis is rarely sought. Our case exemplifies the typically occult presentation. ${ }^{5}$ Common cerebral symptoms of meningeal carcinomatosis such as headache, lethargy and confusion, and common spinal root involvement presenting as back pain or weakness of extremities are easily ascribed to other illness, medications, bony metastases or systemic effects of cancer and go undiagnosed pre-mortem..$^{5,6}$

Diagnosis of meningeal carcinomatosis always requires a high index of suspicion; in cases of prostate carcinoma, the diagnosis is rarely considered and lumbar punctures are rarely performed because the prostate has been a rarely recognised source of meningeal carcinomatosis. In addition, when CSF is examined, the diagnosis of meningeal carcinomatosis is missed on the initial CSF cytology in up to $55 \%$ of cases. ${ }^{6}$ Finally, diagnoses of meningeal carcinomatosis missed pre-mortem

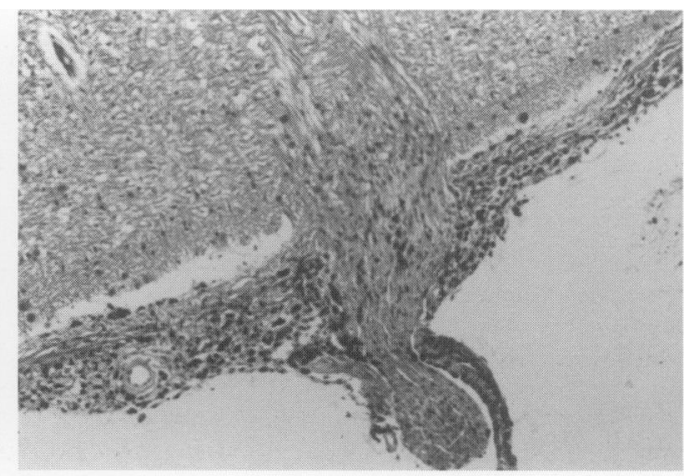

B 


\section{Summary points}

- the potential of prostate carcinoma to produce meningeal carcinomatosis should be recognised by clinicians, who must maintain a high index of suspicion when patients with advanced disease present with cerebral symptoms, back pain, or neurologic findings

- the diagnosis may require repeated CSF cytologic examinations, and immunocytochemical stains should be considered to confirm a prostatic source if adenocarcinoma cells are identified

- androgen ablative therapy may give prolonged remissions in patients with previously untreated disease

are unlikely to be diagnosed at autopsy, because of the typical practice of exempting the brain and spinal cord from post-mortem dissection. While the true incidence of prostatic meningeal carcinomatosis remains unknown, we speculate that the incidence is increasing, as demonstrated for other solid tumours, ${ }^{6}$ due to therapeutic advances that sustain patients with advanced carcinoma for longer periods of time. Our case is the first demonstration that when the diagnosis of

1 Mencel PJ, DeAngelis LM, Motzer RJ. Hormonal ablation as effective therapy for carcinomatous meningitis from prostatic carcinoma. Cancer 1994; 73: 1892-4.

2 Mahadevia PS, Kiely TM. Meningeal carcinomatosis secondary to prostatic carcinoma: a case report. $\mathcal{f}$ Urol 1980; 124: 154-5.

3 Ehya H, Hajdu SI, Melamed MR. Cytopathology of nonlymphoreticular neoplasms metastatic to the central nervous system. Acta Cytol 1996; 25: 599-609. meningeal carcinomatosis is suspected, a definitive diagnosis of metastatic prostatic adenocarcinoma can be established by cytological examination. In addition to typical histologic characteristics, ${ }^{3}$ prostate-specific markers such as prostate-specific antigen and prostatic acid phosphatase can be identified by immunohistochemistry. Thus, when meningeal carcinomatosis is the first presentation of disseminated prostate adenocarcinoma, ${ }^{2}$ these immunocytologic stains can be used to identify the primary source of tumour.

Meningeal carcinomatosis from a variety of tumours have been treated with intrathecal or intraventricular chemotherapy and radiation therapy. ${ }^{3,4,6}$ Although prostate cancer is largely refractory to chemotherapy, radiation therapy may be effective for treating localised foci of metastases to the leptomeninges. ${ }^{1}$ In addition, hormonal ablation therapy has been reported in one case of previously untreated prostate carcinoma to produce complete neurologic recovery and to limit progression of leptomeningeal tumour. ${ }^{1}$ Whether additional hormonal manipulation can favourably influence tumour progression in patients such as ours who are on chronic leuprolide therapy is not known.

4 Herman DL, Courville CB. Pathogenesis of meningeal carcinomatosis: report of case secondary to carcinoma of cecum via perineural extension; with a review of 146 cases Bull LA Neurol Soc 1965; 30: 107-17.

5 Olson Me, Chernik NL, Posner JB. Infiltration of the leptomeninges by systemic cancer: a clinical and pathologic study. Arch Neurol 1974; 30: 122-37.

6 Grossman SA, Moynihan TJ. Neoplastic meningits. Neurol Clin 1991; 9: 843-56. 